\title{
Detection of Biofilm Formation in Multi-Drug Resistant Staphylococcus aureus from Rural Tertiary Care Hospital in North India
}

\author{
Chinithung Ngullie*, Ashma Khatun, Varsha A. Singh, Nitasha and Rajdeep Paul \\ Department of Microbiology, MMIMSR, Mullana, Ambala, Haryana, 133207, India \\ *Corresponding author
}

\begin{tabular}{|l|}
\hline K e y w o r d s \\
Biofilm, \\
$\begin{array}{l}\text { Multi-drug resistant } \\
\text { Staphylococcus } \\
\text { aureus. }\end{array}$ \\
\hline Article Info \\
\hline $\begin{array}{l}\text { Accepted: } \\
\text { 07 September } 2017 \\
\text { Available Online: } \\
\text { 10 October } 2017\end{array}$ \\
\hline
\end{tabular}

A B S T R A C T
One of the hallmark characteristic of Staphylococcus aureus infection is its inherent ability to form biofilm on abiotic and abiotic surfaces. Biofilm producing Organisms are notoriously hard to treat owing to their high resistance against commonly used antibiotics. This study aimed at Detection of biofilm formation by Multi-drug resistant Staphylococcus aureus strain resistant to methicillin and Ciprofloxacin from clinical samples. The objectives of the study were: To detect biofilm formation by multi-drug resistant Staphylococcus aureus isolated from clinical specimens by three different methods: Tissue Culture Plate (TCP), Tube Adherence Method (TAM) and Modified Congo Red Agar (MCRA) method. The main objective is to determine the sensitivity, specificity, positive predictive value and negative predictive value of MCRA and TAM against TCP method. A total of 50 Staphylococcus aureus strains resistance to methicillin and Ciprofloxacin from all clinical isolates from April 2015 to March 2016 was subjected to biofilm detection methods. Out of $50 \mathrm{~S}$. aureus resistant to methicillin and ciprofloxacin, 26(52\%) were found to be biofilm producers. Rate of Detection of biofilm was highest by Modified Congo red agar (42\%) and Tube Adherence method (38\%) followed by Tissue Culture plate method (32\%). Rate of detection by three different methods in combination was $42.3 \%$ and decreased as per number of tests. In the present study the rate of biofilm producers detected by all three methods was 26(52\%). Among which, Modified Congo Red Agar Method was best 21(42\%) followed by Tube adherence method 19(38\%) and Tissue Culture Plate Method 16 (32\%). Rate of detection by combination of all three methods was $52 \%$. Hence, this study endorses for frequent detection of Biofilm production by $S$. aureus especially by multi-drug resistant organisms.

\section{Introduction}

Prevalence of Staphylococcus aureus infection is the one that worries many countries, especially when it becomes a human life threatening disease, currently this pathogen is endemic in many countries worldwide. ${ }^{1}$ the hallmark characteristic of S. aureus is its inherent ability to form biofilm on biotic and abiotic surface consequently reducing the antibiotic diffusion rates leading to antibiotic resistance and making them notoriously hard to treat. ${ }^{2}$ As the entrance of certain types of immune barricades like macrophages, shows incomplete penetration into the biofilm medium organisms growing in it easily continue to exist. ${ }^{3}$ Therefore, it is essential to detect biofilm as early as possible as it may help in appropriate treatment. Various approaches to detect biofilm 
comprise assessing visually by electron microscopy and genotypic methods such as Polymerase chain reaction which is costly and beyond the reach of poor settings laboratories. The phenotypic techniques such as Tissue culture plate (TCP) method which is quantitative test, which is considered as a gold standard method, Tube method (TM), Congo red agar method, Modified Congo Red Agar method (MCRA) can be used in routine laboratories test.

\section{Materials and Methods}

A cross sectional study was conducted on 294 clinical specimens in the Department of Microbiology M. M. Institute of Medical Sciences and research Mullana, AMBALA from April 2015 to March 2016. Multi-drug resistant (resistant to methicillin and ciprofloxacin) $S$. aureus strains were selected and biofilm formation was detected by:

\section{Tissue Culture Plate Method (TCPM)}

Test strains were inoculated in brain heart infusion broth (BHI) with $2 \%$ sucrose, incubated for $24 \mathrm{hrs}$ at $37^{\circ} \mathrm{C}$. On the next day $200 \mu \mathrm{l}$ inoculum from BHI broth was filled in microtiter well and again incubated for $24 \mathrm{hrs}$ at $37^{\circ} \mathrm{C}$. The microtiter plate was washed four times with $0.2 \mathrm{ml}$ of phosphate buffer saline (PBS pH 7.2) and fixed with sodium acetate $(2 \%)$. It was stained with crystal violet $(0.1 \% \mathrm{w} / \mathrm{v})$ for one minute. Then rinsed off with deionized water and dried.

The biofilm if present on all side wells were uniformly stained with crystal violet. Optical Density (OD) of stained adherent bacteria was determined with a micro ELISA auto analyser at wavelength of $570 \mathrm{~nm}(\mathrm{OD} 570 \mathrm{~nm})$. These OD values was considered as index of bacteria adhering to surface and forming biofilms. The experiment was repeated three times and the data was then averaged to calculate the standard deviation. The biofilm formation, due to adherence of bacteria was classified based on OD values as follows:

\section{Tube Adherence Method (TAM)}

Test strains were inoculated in brain heart infusion broth (BHI) with $2 \%$ sucrose and incubated for $24 \mathrm{hrs}$ at $37^{\circ} \mathrm{C}$ in a test tube. The tube was washed for four times with $0.2 \mathrm{ml}$ of phosphate buffer saline (PBS $\mathrm{pH}$ 7.2) and stained with crystal violet $(0.1 \% \mathrm{w} / \mathrm{v})$ for one minute. The excess stain was removed by washing it with distilled water for three times. Dry tubes were kept in inverted position, the biofilm was seen at bottom of the tube and along the side of walls of test tube.

\section{Modified Congo Red Agar method (MCRA)}

Test strains were inoculated on Modified Congo red agar, Incubated for 24-48 hours at $37^{\circ} \mathrm{C}$.Black colored colonies with dry crystalline consistency were interpreted as positive biofilm producing strains whereas red colored colonies as negative.

\section{Results and Discussion}

A total of 294 samples including blood, pus, urine, wound, sputum, ear discharge HVS and Foley tip were taken in which the rate of Staphylococcus aureus isolates was $25.85 \%$ out of which $65.7 \%$ showed multi-drug resistance. $52 \%$ strains were biofilm producers by all the three phenotypic methods (Table 1).

The detection of biofilm production was highest by Modified Congo red agar (42\%) followed by Tube Adherence method (38\%) and Tissue Culture plate method (32\%) (Table 2 and Figs. 1-3). Interestingly, the biofilm formation detected was highest by combination of all three methods $42.3 \%$ while 
MCRA+TAM detected $15.4 \%, 11.5 \%$ by MCRA+TCP and $7.6 \%$ by TAM+TCP (Table 3 ). Multidrug resistant $S$. aureus is still one of the five most common causes of nosocomial infections conferring it almost impossible to be eradicated if associated with biofilm production. The prevalence of biofilm formation varies from hospital to hospital ${ }^{4}$.
On the same grounds, In the present study, rate of biofilm producing Multi-drug resistant Staphylococcus aureus came out to be $52 \%$.(Table 1) which was in well accordance with the studies done by Jayashri Apurva et al., ${ }^{5}$, Sibabrata et al., ${ }^{6}$ and Richa Gupta $e t$ al., ${ }^{7} 52.63 \%, 53.2 \%$ and $54.5 \%$ respectively.

Fig.1 Modified Congo Agar method for detection of biofilm

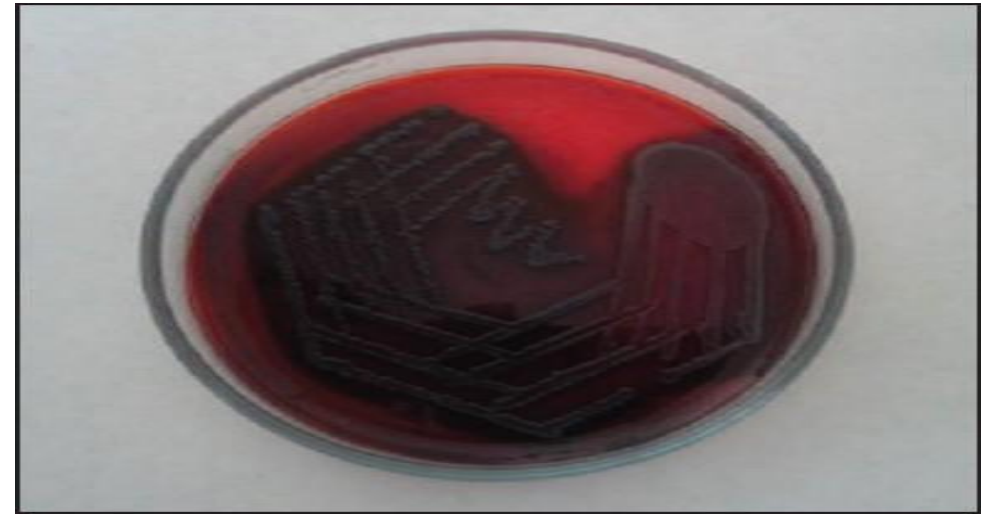

Fig.2 Tube Adherance method for detection of biofilm

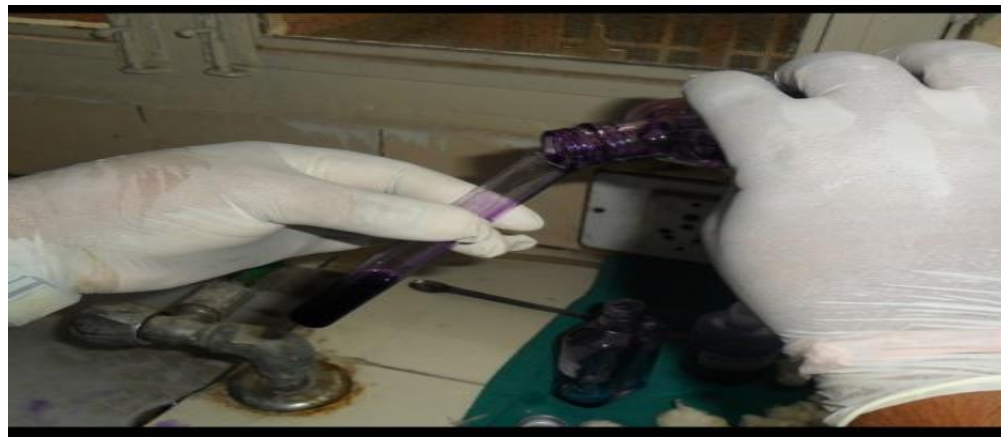

Fig.3 Tissue Culture Plate method for detection of biofilm

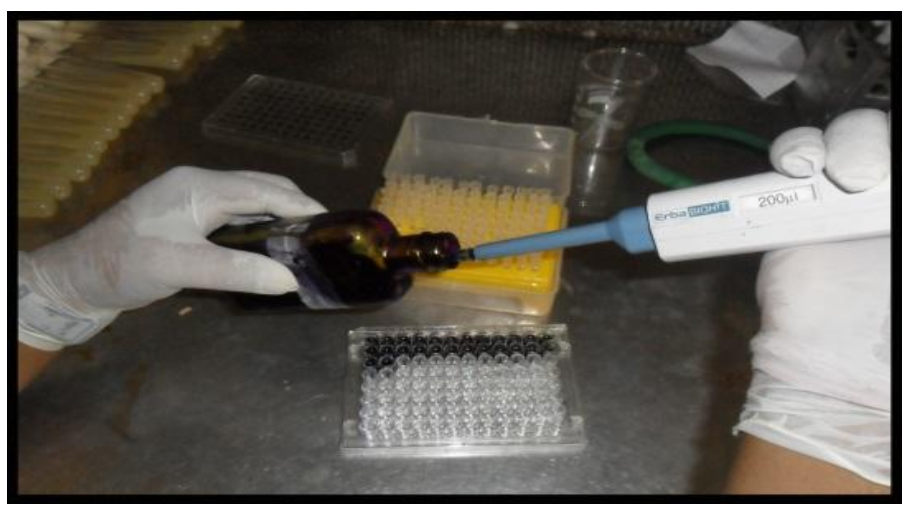


Table.1 Rate of biofilm producing multidrug resistance Staphylococcus aureus

\begin{tabular}{|l|l|}
\hline $\begin{array}{l}\text { Multi drug resistance } \\
\text { Staphylococcus aureus }\end{array}$ & $\begin{array}{l}\text { Biofilm producers } \\
\text { (by all three methods) }\end{array}$ \\
\hline 50 & $26(52 \%)$ \\
\hline
\end{tabular}

Table.2 Rate of Detection of biofilm production by individual phenotypic methods

\begin{tabular}{|l|l|l|}
\hline Methods & Number of biofilm producers & Percentage of biofilm producers \\
\hline Tissue culture plate method & 16 & $32 \%$ \\
\hline Modified Congo red agar method & 21 & $42 \%$ \\
\hline Tube adherence method & 19 & $38 \%$ \\
\hline
\end{tabular}

Table.3 Rate of biofilm production by three methods in combination

\begin{tabular}{|l|l|l|}
\hline $\begin{array}{l}\text { Total Number of Positive } \\
\text { Strains By N=26 }\end{array}$ & Total number of isolates & $\begin{array}{l}\text { Total percentage of } \\
\text { isolates }\end{array}$ \\
\hline MCRA + TAM + TCP & 11 & $42.3 \%$ \\
\hline MCRA + TAM & 4 & $15.4 \%$ \\
\hline MCRA + TCP & 3 & $11.5 \%$ \\
\hline TAM + TCP & 2 & $7.6 \%$ \\
\hline
\end{tabular}

Tissue Culture Plate Method (TCPM)

\begin{tabular}{|l|l|l|}
\hline Mean OD Value & Adherence & Biofilm formation \\
\hline$<0.120$ & Non & Non/Weak \\
\hline $0.120-0.240$ & Moderate & Moderate \\
\hline 0.240 & Strong & High \\
\hline
\end{tabular}

The rate of detection of multidrug resistant Staphylococcus aureus in current study was maximum by Modified Congo red agar Method, $42 \%$, followed by tube adherence method $38 \%$ and lowest positivity $32 \%$, was rendered by TCP method (Table 2). The sensitivity, specificity and overall accuracy of MCRA was $82.35 \%, 75.75 \%, 78 \%$ and of Tube method $82.35 \%, 84.84 \%, 84 \%$.

It was further supported by the studies done by Rewatkar et al., ${ }^{8}$ and Wenbo Hou et al., 9 by Congo Red Agar Method, 45\% and $55.56 \%$ respectively. The current study was totally contradictory to the study of Neetu Peedikayil John et al., ${ }^{10}$ in which Congo Red Agar gives the lowest positivity, $4.30 \%$. Many researchers have reported that tissue culture plate method is considered to be the gold standard for the biofilm detection as it provides only true positive results due to hydrophobic interaction between two surfaces, bacterial surface and substratum. Despite this fact, the current study showed the maximum detection of biofilm producers by MCRA since this method is fast, reproducible, and evaluation criteria is based on visual analysis of the color of colony.

Furthermore, in TAM, strong biofilm producers could be easily detected but it was difficult to discriminate between weak and biofilm negative isolates as results may vary by different observer. The best result observed was by combination of all three methods than a single one. In the present 
study, the results with combination of all three methods showed $42.3 \%$ (Table 3) positivity followed by the rate was decreasing as the less number of tests were evaluated. As far as our knowledge is concerned this is the first study which has shown the results in combination.

To conclude Study was conducted in the department of microbiology on 294 various clinical samples. The rate of biofilm production was $52 \%$. The biofilm detection was maximum by modified Congo red agar method (42\%), followed by Tube adherence method (38\%) and Tissue culture plate method $(32 \%)$. Detection of biofilm by combination of all three methods showed $42.3 \%$. Therefore early detection of Biofilm production of multi-drug resistant Staphylococcus aureus by these tests should be included in routine to minimized the risk of infection especially among the patients who are admitted in hospital, thereby enabling quick treatment and reducing the mortality and morbidity rate effectively. For the optimum result the analysis should always be done by combination of all three methods.

\section{References}

1. Ismaeel H Bozakouk, Najla Mustafa El Hassi, Nadia El Megrab et al. Community associated Staphylococcus aureus infections among children at the paediatric hospital in Benghazi city/ Libya.Int.J.Curr.Microbiol.App.Sci .2014; 3(5): 634-639.

2. Donlan RM, Costerton JW. Biofilms: survival mech- anisms of clinically relevant microorganisms. Clin Microbiol
Rev 2002; 15:167-93; PMID:11932229; DOI:10.1128/CMR.15.2.167-93.2002

3. Jessica L. Lister and Alexander R. Horswill. Staphylococcus aureus biofilms:Recent developments in biofilm dispersal. Front. Cell. Infect. Microbiol., 2014;1-16.

4. Patel R. Biofilms and antimicrobial resistance. Clin Orthop Relat Res. 2005; (437):41-47

5. Apurva $\mathbf{J}$ et al.Biofilm Forming Abilities and Antibiotic Susceptibility Pattern of Clinical Isolates of Staphylococcus aureus. IJAR.2013; Volume $3: 41-44$

6. Bhattacharya S.Evaluation of multidrug resistant Staphylococcus aureus and their association with biofilm production in a tertiary care hospital,Tripura,Northeast india. Journal of Clinical and Diagnostic Research. 2015 Sep, Vol-9(9): 1-4

7. Gupta R,Malik A, Rizvi M, Ahmed M and Hashmi A. Multidrug Resistant Gram Positive Pathogens with Special Reference to MRSA and Biofilm Production in ICU Patients: Recurrent Challenge for Clinicians. Int. J. Curr. Microbiol. App. Sci (2015) Special Issue-1: 207-212.

8. R. Rewatkar, Dr. B. J. Wadher. Staphylococcus aureus and Pseudomonas aeruginosa-Biofilm formation methods. IOSR-JPBS.2013;Volume 8( 5 ):PP 36-40.

9. Wenbo Houeta. Biofilm Forming Capacity of Staphylococcus epidermidis, Staphylococcus aureus and Pseudomonas aeruginosa from Ocular Infections. IOVS.2012; vol53:5624-5631.

10. Neetu peedikayil john et al.Biofilm formation by methicillin resistant S.aureus and their antibiotic susceptibility pattern:An in vitro study.2014;7(1):1-11.

\section{How to cite this article:}

Chinithung Ngullie, Ashma Khatun, Varsha A. Singh, Nitasha and Rajdeep Paul. 2017. Detection of Biofilm Formation in Multi-Drug Resistant Staphylococcus aureus from Rural Tertiary Care Hospital in North India. Int.J.Curr.Microbiol.App.Sci. 6(10): 522-526. doi: https://doi.org/10.20546/ijcmas.2017.610.063 